\title{
Hyponatremia: How to Approach This Confusing Abnormality
}

The syndrome of inappropriate secretion of antidiuretic hormone (SIADH) is not so frequent but it is a representative entity of hyponatremia. Although the clinical features were established by Bartter and Schwartz more than 30 years ago (1), a prototype of their diagnostic criteria has been applied so far. Hyponatremia is not a rare condition, especially in elderly persons. Nearly 1 to $5 \%$ of hospital patients showed hyponatremia, defined as a serum sodium level of less than 130 $\mathrm{mEq} / l(2,3)$, therefore, the appropriate differential diagnosis is essential. Hyponatremia is generally divided into two different pathophysiological states, hypervolemic and hypovolemic hyponatremia. SIADH is usually classified as normovolemic hyponatremia according to standard clinical assessment of extracellular fluid (ECF) volume. However, strictly speaking, a mild but apparent increase in ECF volume is present which then induces dilutional hyponatremia. In clinical practice, the differentiation of these two states is important because the treatments are quite varied. Generally, water restriction is the first therapeutic choice in hypervolemic hyponatremia including SIADH, on the other hand, water deprivation aggravates the symptoms of patients with hypovolemic hyponatremia. The concept of SIADH is very familiar to physicians, thus the syndrome is readily suspected when patients with hyponatremia are encountered. There are many conditions which resemble SIADH. For instance, cerebral salt wasting syndrome (CSWS) is often seen as hyponatremia after neurosurgery (4). SIADH also occurs due to brain tumor or cerebrovascular accident. Both conditions show hyponatremia, urinary sodium loss and no signs of edema or thyroidal and adrenal insufficiency. However, the plasma volume is contracted in the case of CSWS, suggesting that water restriction as a treatment for hyponatremia should be contraindication. Hypovolemia in CSWS per se is a potent stimulating factor for antidiuretic hormone, vasopressin, secretion in even the hypo-osmolar state and it is also well known that patients with SIADH show a detectable level of plasma vasopressin. Accordingly, the plasma vasopressin concentration does not have a positive role in the differential diagnosis of these hyponatremic states. Then, what is an effective and practical tool for differentiation of hyponatremia? Unfortunately, we do not have a simple efficient method for the evaluation of ECF or plasma volume, however, the hypervolemic state usually indicates that the plasma renin concentration or serum uric acid is low or relatively low in contrast with hypovolemic hyponatremia.

The etiology of SIADH is markedly varied, including diseases in the central nervous system or pulmonary region which may cause a disturbance in the control system of vasopressin secretion. Other mechanisms to manifest SIADH are vaso- pressin producing tumors or some kinds of medications. Small cell lung cancer is the most possible candidate as an ectopic vasopressin production. Vasopressin is autonomously produced from cancer cells even in the extremely low plasma osmolality and no suppression occurs until cancer resection or chemo- or irradiation-therapy for the tumor. Approximately $40 \%$ of small cell lung cancer cases showed inappropriate secretion of vasopressin and $14 \%$ of the patients indicated abnormalities in serum sodium or urine osmolality (5). Many patients with hyponatremia do not present any clinical symptoms, especially mild (>125 mEq/l) or chronic occasion. However, we must not exclude the possibility that some serious disorders may be latent or not in even asymptomatic hyponatremia. Hyponatremia sometimes appears as the first sign of malignant or severe diseases (6).

$$
\text { See also p } 950 \text {. }
$$

It is simple but fundamental to measure serum sodium at regular intervals especially in hospital patients, because hyponatremia-induced symptoms are rather infrequent and obscure. The apparent symptoms usually occur by rapid lowering of serum sodium or chronic hyponatremia of less than 120 $125 \mathrm{mEq} / \mathrm{l}$.

Finally, the treatment of hyponatremia is also complicated. Acute correction of chronic hyponatremia sometimes causes an iatrogenic complication known as myelinolysis or central pontine myelinolysis (7). We must keep in mind that the correction of chronic hyponatremia needs careful consideration and the correction rate should be kept at less than $0.5 \mathrm{mEq} / / / \mathrm{h}$ to prevent myelinolysis.

Yutaka Orso, MD The First Department of Internal Medicine, Nagoya University School of Medicine, Showa-ku, Nagoya 466-8550

\section{References}

1) Bartter FC, Schwartz WB. The syndrome of inappropriate secretion of antidiuretic hormone. Am J Med 42: 790-806, 1967.

2) Anderson RJ, Chung HM, Kluge R, Schrier RW. Hyponatremia: a prospective analysis of its epidemiology and the pathogenetic role of vasopressin. Ann Intern Med 102: 164-168, 1985.

3) Flear CT, Gill GV, Burn J. Hyponatremia: mechanisms and management. Lancet 2 (8236): 26-31, 1981.

4) Harrigan MR. Cerebral salt wasting syndrome: a review. Neurosurgery 38: $152-160,1996$.

5) von Rohr A, Cerny T, Joss RA, Brunner KW. Syndrome of inappropriate $\mathrm{ADH}$ secretion (SIADH) in small-cell bronchus carcinoma. Schweiz 
Med Wochenschr 121: 1271-1282, 1991.

6) Kamoi K, Kurokawa I, Kasai H, et al. Asymptomatic hyponatremia due to inappropriate secretion of antidiuretic hormone as the first sign of a small cell lung cancer in an elderly man. Intern Med 37: 950-954, 1998.

7) Laureno R, Karp BI. Myelinolysis after correction of hyponatremia. Ann Intern Med 126: 57-62, 1997. 